\title{
Spectroscopy of Charmed and Bottom Hadrons using Lattice QCD
}

\author{
Sourav Mondal ${ }^{1}$, M. Padmanath ${ }^{2}$, and Nilmani Mathur ${ }^{1, \star}$ \\ ${ }^{1}$ Department of Theoretical Physics, Tata Institute of Fundamental Research, Mumbai. \\ ${ }^{2}$ Institüt fur Theoretische Physik, Universität Regensburg, \\ Universitässtrase 31, 93053 Regensburg, Germany.
}

\begin{abstract}
We present preliminary results on the light, charmed and bottom baryon spectra using overlap valence quarks on the background of $2+1+1$ flavours HISQ gauge configurations of the MILC collaboration. These calculations are performed on three different gauge ensembles at three lattice spacings $(a \sim 0.12 \mathrm{fm}, 0.09 \mathrm{fm}$ and $0.06 \mathrm{fm})$ and for physical strange, charm and bottom quark masses. The SU(2) heavy baryon chiral perturbation theory is used to extrapolate baryon masses to the physical pion mass and the continuum limit extrapolations are also performed. Our results are consistent with the well measured charmed baryons. We predict the masses of many other states which are yet to be discovered.
\end{abstract}

\section{Introduction}

Energy spectra of heavy hadrons as well as the spin splittings between them plays an important role in theoretical understanding of the strong interactions of our universe. With the recent experimental discovery of the doubly charmed baryon, $\Xi_{c c}^{++}$[1], and various $\Omega_{c}^{0}$ resonances [2] by the LHCb collaboration, there has been resurgence of the scientific interest in the study of heavy baryons. In the near future, one expects many more discoveries of new heavy hadrons at ongoing experiments at LHCb, BESIII and future experiments at Belle. On the other hand, the first principle calculations using Lattice QCD is an essential tool to study the energy spectra of these heavy hadrons. First principle calculations using Lattice QCD are essential to study the spectrum of these heavy hadrons in a model independent way. Not only that these calculations can provide crucial inputs for the understanding of the structure and interactions of these states, but also make predictions that can guide future discoveries. However, lattice QCD study of heavy baryons is severely affected by the large discretization error due to relatively larger heavy quark masses. Therefore, it is crucial to take appropriate continuum limit to eliminate this systematic error. In this talk we present updated results from our ongoing study on the hadron spectra. Particular emphasis is given on the calculations of the charm and bottom baryons which are extracted at three lattice spacings and then are extrapolated to the continuum limit.

${ }^{\star}$ Speaker, e-mail: nilmani@ theory.tifr.res.in 


\section{Numerical Details}

We use three sets of dynamical 2+1+1 flavour ensembles generated by the MILC collaboration. The gauge ensembles were generated on lattices of sizes $24^{3} \times 64,32^{3} \times 96$ and $48^{3} \times 144$ at gauge couplings $\beta=6.00,6.30$ and 6.72 respectively. The details of these gauge configurations are summarized in Ref. [3]. We use $\Omega_{s s s}$ baryon to measure lattice spacings [4, 5] and those are found to be consistent with $0.1207(11), 0.0888(8)$ and $0.0582(5) \mathrm{fm}$., as measured by the MILC collaboration using $r_{1}$ parameter.

For the light and charm quarks we use a unified approach adopting overlap fermions, which does not have $O(m a)$ errors and has exact chiral symmetry at finite lattice spacing. Details of the action, its numerical implementation, mass tuning are given in Refs. [4, 5]. The tuned bare charm quark masses $\left(a m_{c}\right)$ are found to be $0.528,0.425$ and 0.29 on coarser to finer lattices respectively [5].

While we intend to treat bottom quarks similarly in future with the same formalism, for the current work we use a non-relativistic formulation [6]. This NRQCD Hamiltonian is improved by including spin-independent terms through $O\left(v^{4}\right)$. For the coarser two ensembles, we use the values of the improvement coefficients, $c_{1}$ to $c_{6}$, as estimated non-perturbatively by the HPQCD collaboration [7]. For the finer lattice, we use tree level coefficients. The details of the NRQCD action and tuning is given in Ref. [8,9]. The spin averaged $1 S$ bottomonium kinetic mass is used to tune the bottom quark mass [8]. The observed hyperfine splitting $(64 \pm 3 \mathrm{MeV})$ in $1 S$ bottomonium is found to be in good agreement with its experimental value $(62.3 \pm 3 \mathrm{MeV})$.

\section{Results}

We have calculated hadron spectra over a wide range of pseudoscalar meson masses from the physical pion mass to pseudoscalar meson mass at about $5.5 \mathrm{GeV}$. On finer lattices pseudoscalar meson mass ranges are from about $300 \mathrm{MeV}$ to around $6 \mathrm{GeV}$. In future we intend to extend pseudoscalar meson mass range towards $\eta_{b}$ on a hyperfine lattice. While that calculation is underway, using non-relativistic bottom quarks we have extended this study to calculate the energy spectra of bottom hadrons. While a comprehensive analysis with existing data set is also underway, here we present our preliminary results on the ground state energy spectra for light as well as heavy hadrons with particular emphasis on charmed and charmed-bottom baryons.

\subsection{Light hadrons}

We first show our results on light hadrons in Figure 1. Figure 1(a) shows pseudoscalar meson masses as a function of quark masses on the coarser lattice $(a \sim 0.1207 \mathrm{fm})$ covering a mass range from the physical pion mass to around $5.5 \mathrm{GeV}$. It is quite encouraging to see that the square of the pseudoscalar meson mass approaches the chiral limit linearly with quark mass (modulo small volume effect). In future we will calculate pseudoscalar meson decay constants over these quark mass ranges. In Figure 1(b) we show the hyperfine splittings between vector $\left(1^{-}\right)$and pseudoscalar $\left(0^{-}\right)$mesons on this wide range of pseudoscalar meson masses. In Figure 1(c) we show the similar hyperfine splittings (in $\mathrm{MeV}$ ) between $\Delta$-baryon $\left(J^{P}=\frac{3}{2}^{+}\right)$and nucleon $\left(J^{P}=\frac{1}{2}^{+}\right)$at three lattice spacings and also over a wide range of pseudoscalar meson masses. Figure 1(d) shows our preliminary results on the ground state spectra of the low lying octet baryons on $32^{3} \times 96$ lattice at lattice spacing $\sim 0.088 \mathrm{fm}$. As expected these baryons coincides at the SU(3) flavour symmetric point and deviate from each other on its both sides. An analysis with the chiral and continuum extrapolation of these baryons is ongoing. 


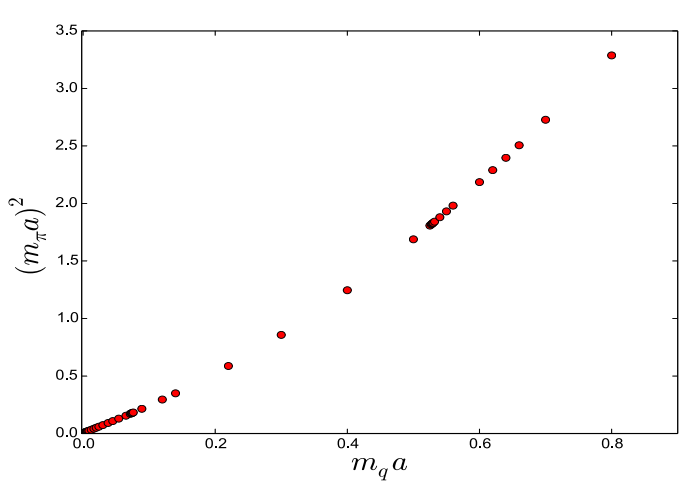

(a)

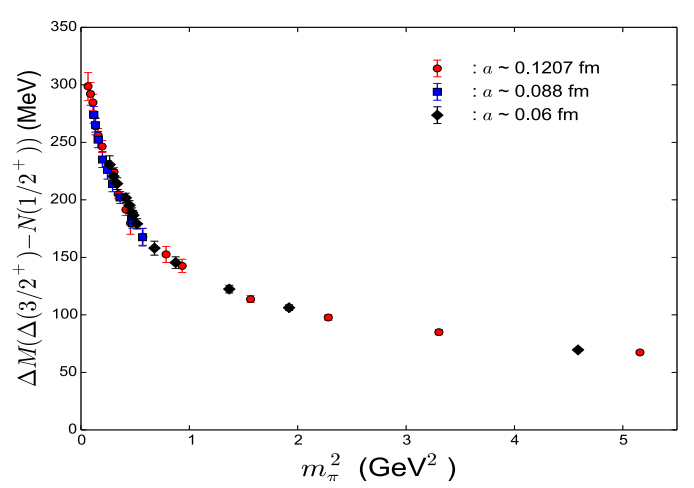

(c)

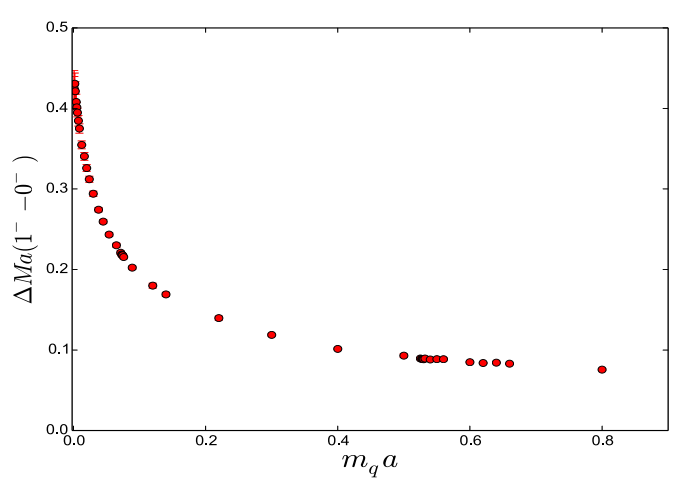

(b)

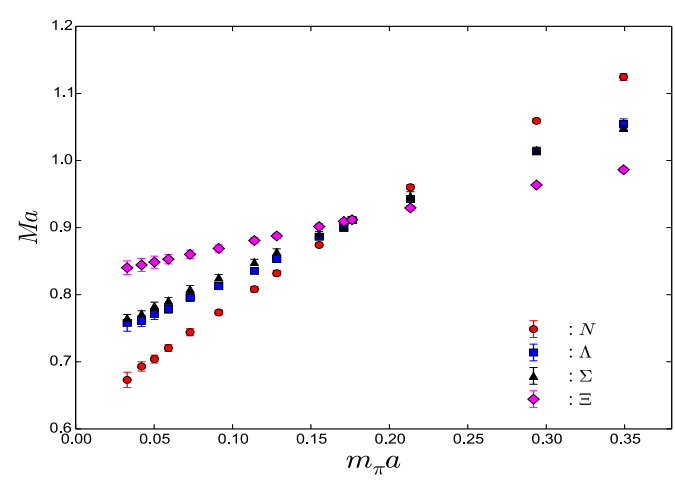

(d)

Figure 1. (a) Square of pseudoscalar meson masses as a function of quark masses from physical pion mass to about $5.5 \mathrm{GeV}$. (b) Hyperfine splittings between vector and pseudoscalar mesons over the same range of pion masses. (c) Hyperfine splittings between $\Delta$-baryon $\left(J^{P}=\frac{3}{2}^{+}\right)$and the nucleon $\left(J^{P}=\frac{1}{2}^{+}\right)$at three lattice spacings and over a wide range of pseudoscalar meson masses. (d) Ground state energy spectra of the lying octet baryon on one finer lattice $(a \sim 0.088 \mathrm{fm})$.

\subsection{Charmed hadrons}

Plethora of experimental discoveries have been made over the last two decades in the heavy hadron sector, part of which are understood theoretically, while the nature of the rest continues to be puzzling [10]. Investigations using first principles such as lattice QCD are crucial to understand the structure and interactions of these excitations as well as to guide future discoveries of more subatomic particles. Being heavy, the energy spectra of heavy hadrons on the lattice are subject to strong discretization errors and thus lattice calculations at more than one lattice spacings followed by the continuum extrapolation is quite essential.

In Figure 2 we plot our preliminary results on continuum extrapolations for charm-strange mesons (Figure 2(a)) and charmonia (Figure 2(b)). To reduce discretization error we calculated splitting of a meson from the respective $1 S$ spin average masses. It is interesting to note that different meson has different slopes towards continuum limit. The continuum extrapolation is carried out using terms up to $O\left(\left(m_{q} a\right)^{3}\right)$ with Bayesian priors (there is no $O\left(m_{q} a\right)$ term for overlap action). We observe that 
except the hyperfine splittings, for other splittings the coefficients for $O\left(\left(m_{q} a\right)^{3}\right)$ terms are very small.

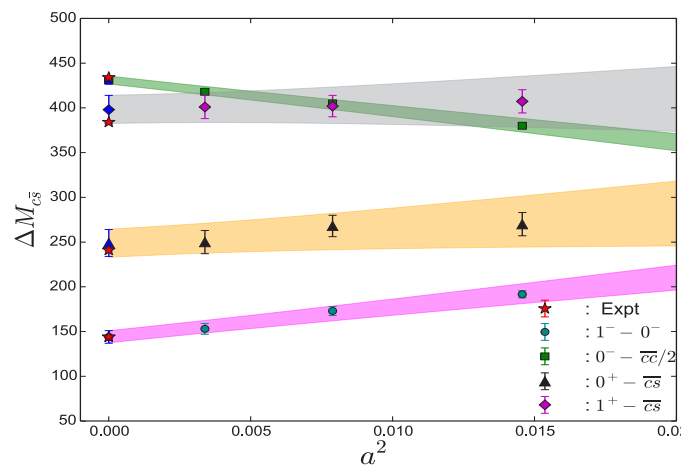

(a)

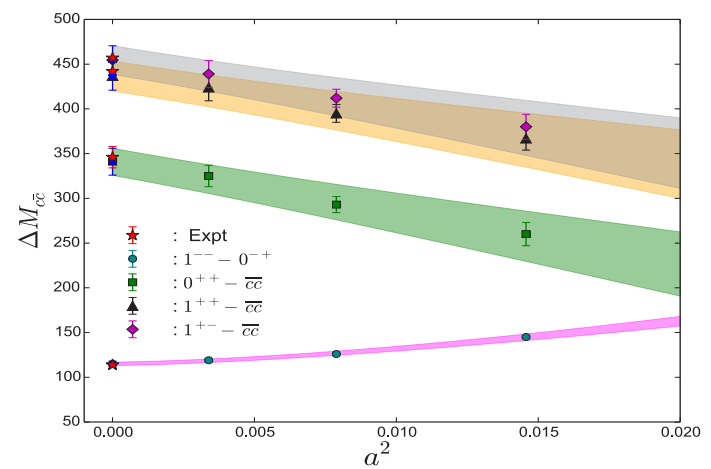

(b)

Figure 2. Hyperfine splittings of $1 S$ quarkonium plotted against the square of the lattice spacings for (a) charmstrange and (b) charmonia at three lattice spacings. Bands represent one-sigma errorbars in the continuum extrapolations.

Next we show our preliminary results for the ground state of the positive parity charmed baryons. In order to reduce discretization error arising out of the tuning of the charm quark mass from the kinetic mass, we first calculate the ratios of baryon masses $\left(M_{b} a\right)$ to the $1 S$ spin-average mass $\left(M_{1 S} a\right)$, i.e., $M_{b}^{r}=M_{b} a / n M_{1 S} a$, where $n=1 / 2$ and 1 for singly- and doubly charmed baryons, respectively. We then perform the chiral extrapolation with a form $M_{b}^{r}=A+B \cdot\left(m_{\pi} a\right)^{2}$. We also utilize the chiral extrapolation form using the heavy baryon chiral perturbation theory (HBChPT), as described in Ref. [11] The HBChPT chiral extrapolation form that we use is the following :

$$
\begin{aligned}
& \frac{m_{\Lambda_{c}}}{m_{\text {spinav. }}}=\frac{m_{\Lambda_{c}}^{0}}{m_{\text {spinav. }}^{0}}+\frac{\sigma_{\Lambda_{c}}}{\left(4 \pi f_{\pi}\right) m_{\text {spinav. }}} m_{\pi}^{2}-\frac{6 g_{3}^{2}}{\left(4 \pi f_{\pi}\right)^{2}\left(m_{\text {spinav. }}\right)}\left(\frac{1}{3} \mathcal{F}\left(m_{\pi}, \Delta_{\Lambda_{c} \Sigma_{c}, \mu}\right)+\frac{2}{3} \mathcal{F}\left(m_{\pi}, \Delta_{\Lambda_{c} \Sigma_{c}^{*}, \mu}\right)\right) \\
& \frac{m_{\Xi_{c}}}{m_{\text {spinav. }}}=\frac{m_{\Xi_{c}}^{0}}{m_{\text {spinav. }}^{0}}+\frac{\sigma_{\Xi_{c}}}{\left(4 \pi f_{\pi}\right) m_{\text {spinav. }}} m_{\pi}^{2}-\frac{3}{2} \frac{g_{3}^{2}}{\left(4 \pi f_{\pi}\right)^{2}\left(m_{\text {spinav. }}\right)}\left(\frac{1}{3} \mathcal{F}\left(m_{\pi}, \Delta_{\Xi_{c} \Xi_{c}^{\prime}, \mu}\right)+\frac{2}{3} \mathcal{F}\left(m_{\pi}, \Delta_{\Xi_{c} \Xi_{c}^{*}, \mu}\right)\right)
\end{aligned}
$$

for $\Lambda_{c}$ and $\Xi_{c}$ respectively. The chiral function $\mathcal{F}$ in eqn. 2 is defined as,

$$
\mathcal{F}(m, \Delta, \mu)=\left(\Delta^{2}-m^{2}+i \epsilon\right)^{3 / 2} \ln \left(\frac{\Delta+\sqrt{\Delta^{2}-m^{2}+i \epsilon}}{\Delta-\sqrt{\Delta^{2}-m^{2}+i \epsilon}}\right)-\frac{3}{2} \Delta m^{2} \ln \left(\frac{m^{2}}{\mu^{2}}\right)-\Delta^{3} \ln \left(\frac{4 \Delta^{2}}{m^{2}}\right),
$$

with $\mathcal{F}(m, 0, \mu)=\pi m_{\pi}^{3}$. Splittings $\Delta$ used in the extrapolation formula are obtained by extrapolating the splittings between two baryons to the physical pion masses using trivial extrapolation form

$$
\Delta_{i j}=\Delta_{i j}^{0}+A \cdot\left(m_{\pi} a\right)^{2},
$$

where $i$ and $j$ are the baryons for which splittings are considered. For $\Lambda_{c}$ and $\Xi_{c}$ we could use HBChPT with $\chi^{2} / d o f \sim 1$. After the chiral extrapolation we perform continuum extrapolation of the chirally extrapolated ratios with a form up to $O\left(a^{2}\right)$ terms. Finally to obtain the physical values we multiply the 
extrapolated values by $n M_{p h y}(1 S: c \bar{c})$. In Figure 3 we show our preliminary continuum extrapolated results for the ground states of singly charm baryons. We compare our results with experimental values of these baryons [10] and also with other lattice results [11-15].

Here we would like to point out that the LHCb Collaboration has recently reported observation of five new resonances based on the invariant mass distribution of $\Xi_{c}^{+} K^{-}$in the energy range between 3000-3120 MeV [2]. These resonances have been interpreted as the excited states of $\Omega_{c}^{0}$ baryon. Before the discovery of these resonances we studied the excited state spectra of $\Omega_{c}^{0}$ baryons in details [16-18]. It is quite satisfying to see that our prediction matches very well with the experimental results and strongly indicates that the observed states $\Omega_{c}(3000)^{0}$ and $\Omega_{c}(3050)^{0}$ have spin-parity $J^{P}=1 / 2^{-}$, the states $\Omega_{c}(3066)^{0}$ and $\Omega_{c}(3090)^{0}$ have $J^{P}=3 / 2^{-}$, whereas $\Omega_{c}(3119)^{0}$ is possibly a $5 / 2^{-}$state [19]. This identification is crucial to decipher the structures of these resonances and as such provide information about the binding of charm resonances through strong interactions.

In Figure 4 we show our preliminary results for doubly charmed baryons. It is noteworthy to point out that all lattice results including the current work are predications before the experimental discovery of $\Xi_{c c}^{++}$by the LHCb collaboration [1]. So far $\Xi_{c c}^{++}$is the only doubly charmed baryon discovered experimentally. In that context lattice predictions for other doubly charmed baryons are very interesting for their future discovery.

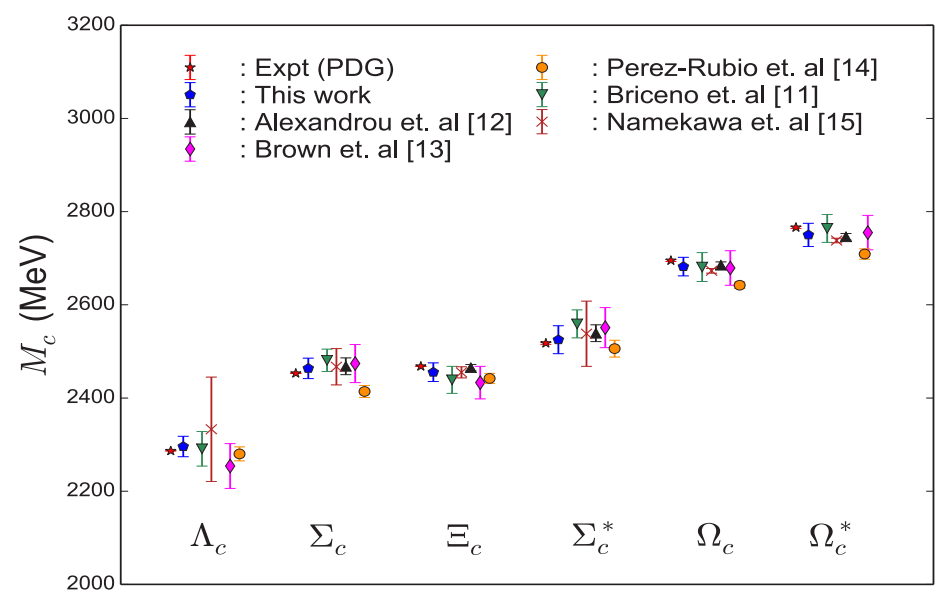

Figure 3. Preliminary results for the positive parity singly charmed baryons. Results from this work are compared with experimental values [10] and other lattice results [11-15].

\subsection{Charmed Bottom Baryons}

After the discovery of the first doubly charmed baryon $\left(\Xi_{c c}^{++}\right)$by the LHCb collaboration [1], presumably the next discovery on doubly heavy baryons may come in the sector of charmed-bottom $(b c)$ baryons. It is to be noted that except the pseudoscalar $B_{c}$ and $B_{c}(2 S)$ mesons no other hadron with $b$ and $c$ quark content has been discovered yet. One would thus expect the discovery of more $b c$ hadrons in near future. We already predicted hyperfine splitting in $B_{c}$ meson to be $55 \pm 4 \mathrm{MeV}$ [8] which constrains the mass of the vector $B_{c}^{*}$ meson.

As mentioned earlier, for the bottom quark we utilize an NRQCD action where the coefficients of NRQCD Hamiltonian are improved up to $O\left(v^{4}\right)$. For the calculation of $b c$ hadrons we use NRQCD 


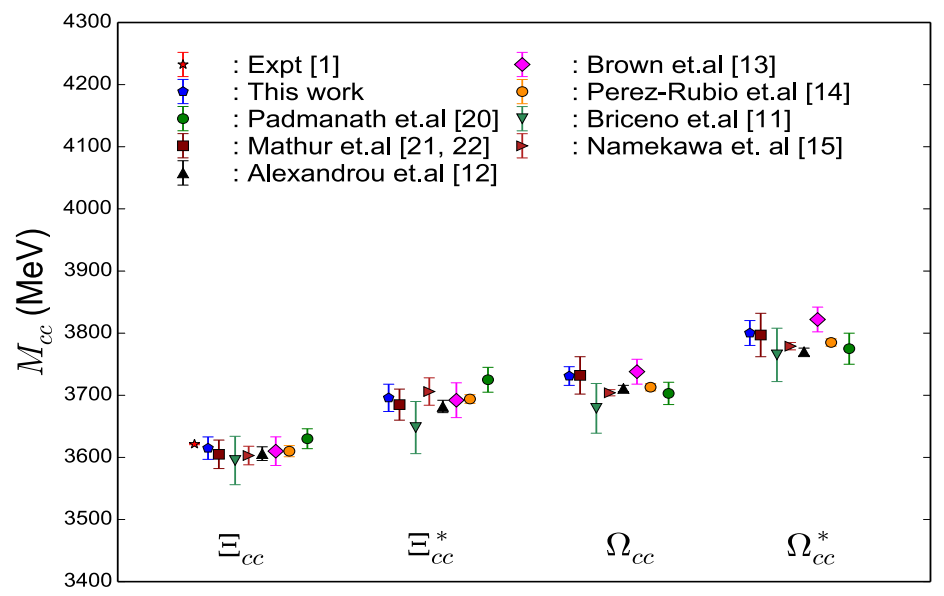

Figure 4. Preliminary results for the positive parity doubly charmed baryons. Results from this work are compared with experimental values (where available) and other lattice results [11-15, 20-22]

propagators for bottom quarks which are contracted with overlap propagators to obtain correlators for various $b c$ hadrons. Here we present the preliminary results on the ground state spectrum of the positive parity charmed bottom $\Omega$ baryons, namely, $\Omega_{c c b}\left(\frac{1}{2}^{+}\right), \Omega_{c c b}^{*}\left(\frac{3}{2}^{+}\right), \Omega_{c b b}\left(\frac{1}{2}^{+}\right)$and $\Omega_{c b b}^{*}\left(\frac{3}{2}^{+}\right)$. Works on the spectra of $\Xi_{c b u}$ and $\Xi_{c b s}$ baryons as well on negative parity baryons are ongoing.

To reduce the relative discretization errors due to charm and bottom quarks we use subtracted mass for the $b c$ baryons. For these baryons we define the subtracted mass as : $M_{\text {sub }} a=M a-$ $n_{c} \overline{M a}(c \bar{c})-n_{b} \overline{M a}(b \bar{b})$, where $\overline{M a}(c \bar{c})$ and $\overline{M a}(b \bar{b})$ are spin-average masses of $1 \mathrm{~S}$ charmonia and bottomonia respectively, whereas $n_{c}$ and $n_{b}$ are the number of charm and bottom quarks in $b c$ hadrons. One would expect that these subtractions will effectively remove the heavy quark content and so will reduce the discretization errors. In Figures 5 we show subtracted energy levels for the charmed-bottom $\Omega$ baryons at two lattice spacings. Our results are compared with the those obtained in Ref. [13]. Horizontal bars are possible errors one may expect to get after continuum extrapolation of hadron masses with non-relativistic bottom quarks. In order to get physical values we need to add back physical value of $n_{c} \bar{M}(c \bar{c})+n_{b} \bar{M}(b \bar{b})$ to above subtracted masses. In future we will add another lattice spacing and then will do continuum extrapolation to obtain final numbers for these baryons.

\section{Conclusions}

We report preliminary results on the ground state energy spectra for various hadrons with light to bottom quark content. We incorporate a unified approach to treat light to charm quarks uniformly using overlap fermions. A relativistic overlap action is used on the back ground of $2+1+1$ HISQ configurations corresponding to lattice spacings of about $0.1207,0.088$ and $0.06 \mathrm{fm}$. Overlap quark propagators are generated using a multimass algorithm over a wide range of quark masses corresponding to pseudoscalar mass from physical pion to about $5.5 \mathrm{GeV}$. In future we will increase this limit towards $\eta_{b}$ using a hyperfine lattice to treat light to bottom quarks with the same action. In this calculation for bottom quark we use a non-relativistic action with non-perturbatively tuned coefficients with terms up to $O\left(v^{4}\right)$. For the coarser two ensembles, we use the values of the improvement coefficients, $c_{1}$ to $c_{6}$, as estimated non-perturbatively by the HPQCD collaboration [18], while for the finer ensemble, 


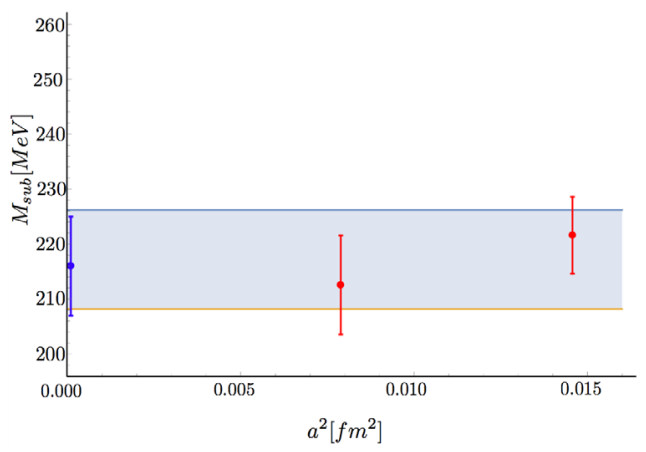

(a) $\Omega_{c c b}$

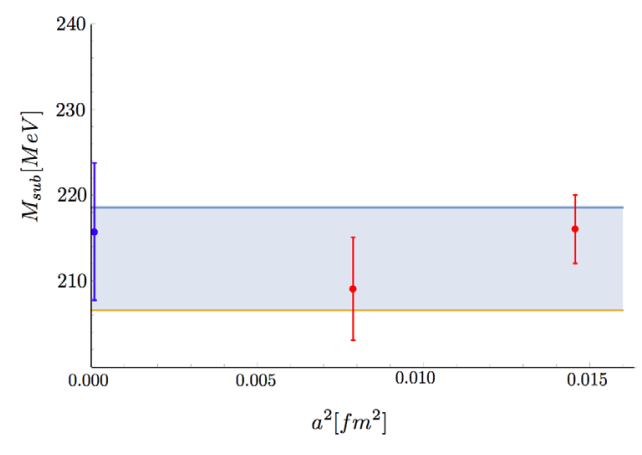

(c) $\Omega_{c b b}$

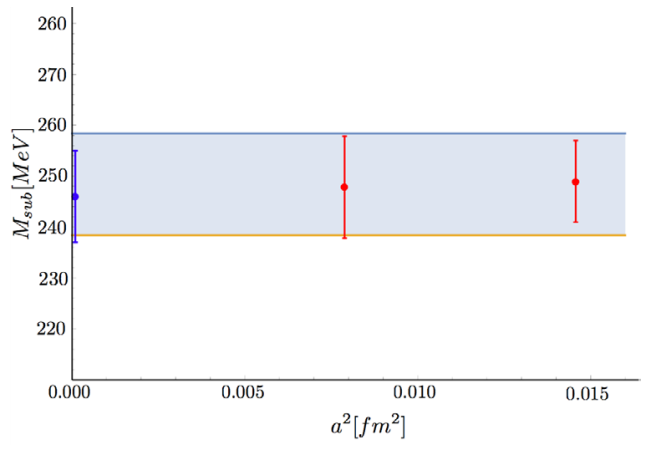

(b) $\Omega_{c c b}^{*}$

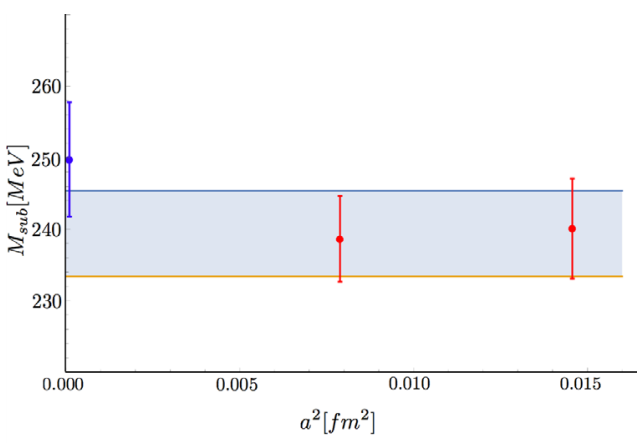

(d) $\Omega_{c b b}^{*}$

Figure 5. Subtracted masses for the charmed bottom $\Omega$ baryons. The red points are the preliminary results from this work and the blue points are the continuum limit result of Ref. [13]

we use tree level coefficients. The charm and bottom masses are tuned by equating the spin-averaged kinetic masses of the $1 S$ charmonia and bottomonia states, respectively, to their physical values.

We present the hyperfine splittings between vector $\left(1^{-}\right)$to pseudoscalar $\left(0^{-}\right)$mesons as well as between $\Delta$-baryon $\left(\frac{3}{2}^{+}\right)$to nucleon $\left(\frac{1}{2}^{+}\right)$in a large range of quark masses. In future we will fit these splittings with appropriate formulae to find out their variations at different quark mass ranges. As is well known that these hyperfine splittings provide very useful information about the interquark interactions and are invaluable ingredients for any potential model calculation. We also show our preliminary results on octet baryons. In future we will take appropriate chiral and continuum limit to get physical results for these observables. We then report our preliminary results on charmed hadrons. Controlling the discretization error is the predominant challenge for heavy quarks and using results at three lattice spacings we are able to take appropriate continuum limit. Extracted value $(115 \pm 3 \mathrm{MeV})$ of the hyperfine splitting of $1 S$ charmonia agree very well to its physical value $(113.5 \pm 0.5 \mathrm{MeV})$, signifying that the discretization error for charm quark in this calculation is in fact under control. Various mesons in the charm-strange sector and charmonia approach correctly to their physical values after continuum extrapolation. We also show our continuum extrapolated results for the ground state of the positive parity singly and doubly charmed baryons. With the recent discovery of $\Omega_{c}^{0}$ baryons [2] 
and doubly charmed $\Xi_{c c}^{++}$baryon [1], there is a resurgence of interest in the study of heavy baryons. We compare our results with other lattice results and also with the experimental results where they are available. There is an overall agreement with various lattice results for singly charmed baryons and their experimental values. For the doubly charmed baryons, lattice results, including results from this work, predate the discovery of $\Xi_{c c}^{++}$baryons. In that context lattice predictions on the energy spectra of other doubly charmed baryons motivate the ongoing and future investigations of these states. We also report our preliminary results on hadrons containing both charm and bottom quarks. The hyperfine splitting of $B_{c}$ meson is found to be $55 \pm 4 \mathrm{MeV}$. For the $b c$ baryons we present results for $\Omega_{c c b}, \Omega_{c c b}^{*}$, $\Omega_{c b b}$, and $\Omega_{c b b}^{*}$. In future we will address other $b c$ baryons including negative parity baryons.

\section{Acknowledgement}

Computations are carried out using computing resources of the Indian Lattice Gauge Theory Initiative and the Department of Theoretical Physics, TIFR. We thank A. Salve, K. Ghadiali and P. Kulkarni for technical supports. M. P. acknowledges support from Deutsche Forschungsgemeinschaft Grant No. SFB/TRR 55 and EU under grant no. MSCA-IF-EF-ST-744659 (XQCDBaryons). We are grateful to the MILC collaboration and in particular to S. Gottlieb for providing us with the HISQ lattices.

\section{References}

[1] R. Aaij et al. (LHCb), Phys. Rev. Lett. 119, 112001 (2017), 1707.01621

[2] R. Aaij et al. (LHCb), Phys. Rev. Lett. 118, 182001 (2017), 1703.04639

[3] A. Bazavov et al. (MILC), Phys. Rev. D87, 054505 (2013), 1212 . 4768

[4] S. Basak, S. Datta, M. Padmanath, P. Majumdar, N. Mathur, PoS LATTICE2012, 141 (2012), 1211.6277

[5] S. Basak, S. Datta, A.T. Lytle, M. Padmanath, P. Majumdar, N. Mathur, PoS LATTICE2013, 243 (2014), 1312.3050

[6] G.P. Lepage et al., Phys.Rev. D46, 4052 (1992),

[7] R.J. Dowdall et al. (HPQCD), Phys. Rev. D85, 054509 (2012), 1110.6887

[8] N. Mathur, M. Padmanath, R. Lewis, PoS LATTICE2016, 100 (2016), 1611.04085

[9] R. Lewis, R.M. Woloshyn, Phys. Rev. D79, 014502 (2009), 0806. 4783

[10] C. Patrignani et al. (Particle Data Group), Chin. Phys. C40, 100001 (2016)

[11] R.A. Briceno, H.W. Lin, D.R. Bolton, Phys. Rev. D86, 094504 (2012), 1207.3536

[12] C. Alexandrou, C. Kallidonis, Phys. Rev. D96, 034511 (2017), 1704.02647

[13] Z.S. Brown, W. Detmold, S. Meinel, K. Orginos, Phys. Rev. D90, 094507 (2014), 1409.0497

[14] P. Perez-Rubio, S. Collins, G.S. Bali, Phys. Rev. D92, 034504 (2015), 1503.08440

[15] Y. Namekawa et al. (PACS-CS), Phys. Rev. D87, 094512 (2013), 1301.4743

[16] M. Padmanath, R.G. Edwards, N. Mathur, M. Peardon, Proceedings Charm (2013), 1311. 4806

[17] P. Madanagopalan, R.G. Edwards, N. Mathur, M.J. Peardon, PoS LATTICE2014, 084 (2015), 1410. 8791

[18] M. Padmanath, N. Mathur, Proceedings Charm (2015), 1508.07168

[19] M. Padmanath, N. Mathur, Phys. Rev. Lett. 119, 042001 (2017), 1704.00259

[20] M. Padmanath, R.G. Edwards, N. Mathur, M. Peardon, Phys. Rev. D91, 094502 (2015), 1502.01845

[21] N. Mathur, R. Lewis, R.M. Woloshyn, Phys. Rev. D66, 014502 (2002), hep-ph/0203253

[22] R. Lewis, N. Mathur, R.M. Woloshyn, Phys. Rev. D64, 094509 (2001), hep-ph/0107037 